J. Phys. IV France 1 (2020)

$\operatorname{Pr} 1-1$

(C) EDP Sciences, Les Ulis

\title{
GAIA Galaxy Survey: a multi-colour galaxy survey with GAIA
}

\author{
Mattia Vaccari ${ }^{1}$ \\ ${ }^{1}$ Dipartimento di Astronomia and CISAS "Giuseppe Colombo", Università di Padova, Vicolo \\ dell'Osservatorio 2, I-35122, Padova, Italy (e-mail: vaccari@pd.astro.it)
}

\begin{abstract}
The performance expected from a galaxy survey to be carried out with GAIA, the GAIA Galaxy Survey, is outlined. From a statistical model of galaxy number density, size and surface brightness distribution, and from detailed numerical simulations based on real images, it is conservatively estimated that GAIA would be able to detect and observe about 3 million galaxies brighter than $V \simeq 17$ and to provide multi-colour and multiepoch broad-band photometry of these with an end-of-mission angular resolution of $\simeq$ 0.35 arcsecand a photometric accuracy of simeq $0.2 \mathrm{mag} / \operatorname{arcsec}^{2}$ at $\mu_{V}=20 \mathrm{mag} / \mathrm{arcsec}^{2}$. The substantial scientific case for performing such a survey and the additional efforts required in terms of mission preparation, operations and telemetry are also discussed.
\end{abstract}

\section{INTRODUCTION}

Since the very beginning of the feasibility studies, the GAIA mission design was driven by the need of determining the position and the brightness of huge numbers of stars with the uttermost accuracy. During the Concept and Technologt Study completed in July 2000 (see [9] and [2]), however, it clearly emerged that such a star-driven mission could also provide, several socalled by-products which would substantially enrich its already impressive scientific yield. In particular, it was realized that the scientific case for GAIA imaging of high-surface brightness sky regions such as the central regions of nearby galaxies was dramatic.

The issues connected with this opportunity have been addressed in a certain detail in a number of studies (see [10, [11], [8] and [12]), and such observations were included in the mission baseline design as described in [2] under the name of GAIA Galaxy Survey. In this paper the current ideas on the implementation of such a survey and on the expected performance are presented.

In Section 2 the overall strategy for galaxy detection and observation with GAIA is outlined. In Sections 3 and 4 the detection and observation, respectively, are discussed in greater detail and results of dedicated studies are presented. In Section 5 the substantial scientific case for performing such a survey is briefly sketched, and in Section 6 the additional efforts required in terms of mission preparation, operations and telemetry are discussed.

Only the measurement of relatively large enhancements of the surface brightness with respect to the sky background is discussed here, although it is believed that the rather similar issue of the observation of Galactic Nebulae could be addressed along the same lines. The measurement of the surface brightness of the sky background, instead, calls for a substantially different approach, as described in [6]. 


\section{GALAXY DETECTION AND OBSERVATION WITH GAIA}

During its scientific operations, the GAIA satellite will continuously spin about its symmetry axis, and the charges contained in the CCD pixels will correspondingly be shifted along-scan to integrate the image for a longer exposure time, a technique known as Time-Delay Integration (TDI). In order to limit the CCDs' reading frequency and the corresponding readnoise and telemetry rate, a dedicated CCD readout process was devised, consisting in detecting objects as they enter the field of view, determining their position, magnitude and signal-to-noise ratio, and, if the latter exceeds a certain limit, collecting data from regions around such stars only. While this approach was the subject of detailed studies as far as point-like objects were concerned, leading to the definition of the Astro telescopes' focal plane and the CCD binning strategy described e.g. in [5], the process of galaxy detection and observation called for a rather different approach in order to identify and measure faint surface brightness variations. As first suggested in [3], galaxies could be detected in the Astro Sky Mapper (ASM) as an average surface brightness significantly in excess over the local sky background and observed in different colours in the Broad Band Photometer (BBP). This observing strategy preventing from optimally observing stars whenever a galaxy is being observed (which is however less than $1 \%$ percent of the time), it could be implemented in one of the two Astros only.

In order to follow this general idea, one had to optimize:

- the size of the areas over which the average surface brightness and the local sky background values are computed. It is presently envisaged to determine the former through trimmed median filtering of ASM 1 samples of $2 \times 2$ pixels and the latter over a few degrees of scan with the same algorithm. However, the exact method used to determine the local sky background is not of interest for our purposes, since the readnoise is by far the dominant noise source.

- the value for the detection area and $S / N$ limit so that useful data are transmitted to the ground without being swamped in less interesting data from the Milky Way or the zodiacal light. The larger the detection area, the fainter the detection limit can be for objects of constant surface brightness, if the error on the sky background is negligible. On the other hand, the detection area should be small enough that a large number of small objects would not be missed.

- the sampling scheme for galaxy observations, so as to establish a trade-off between angular resolution, readnoise and telemetry. As with stars, a larger sample size yields a smaller error on the (average) surface brightness, lower readnoise and telemetry rate but also a lower angular resolution. This aspect is critical for the observations of galaxies, since their potentially very large angular extension will require in some cases the full readout of CCDs.

In the context of the studies carried out to demonstrate the feasibility of the GAIA Galaxy Survey (summarized in [12]), these questions have been given satisfactory (if subject to further improvements) answers.

\section{DETECTION VIA STATISTICAL FORMULAE}

As for the detection, a statistical model of galaxy number density, size and surface brightness distribution was developed in order to characterize the "typical" galaxy (see 10]). While this model obviously cannot do justice to the well-known strong individuality displayed by many galaxies, it is believed to yield sufficiently reliable results when, as in our case, only 


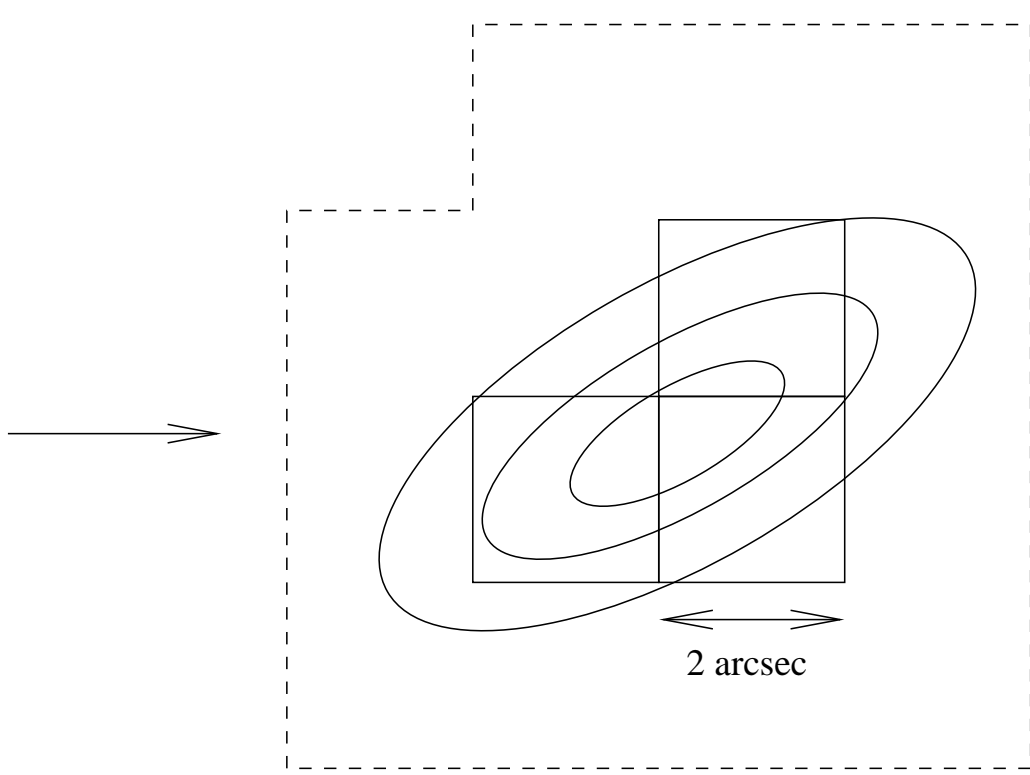

\section{Scan Direction}

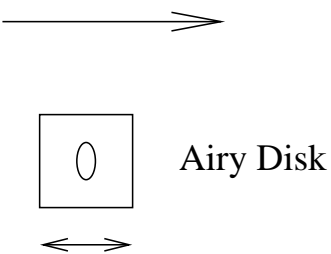

$1 \operatorname{arcsec}$

Fig. 1. Galaxy Detection: a galaxy is detected as an average surface brightness significantly in excess with respect to the sky background over areas of $2 \times 2 \operatorname{arcsec}^{2}$. Data from these areas (solid lines) and from the surrounding regions (dashed line) should then be readout from the CCDs and transmitted to the ground.

statistical properties, i.e. properties averaged over large samples, are of interest. On the basis of such a model, adopting current estimates of GAIA sensitivity and noise, and adapting the statistical formulae for the estimation of the $S / N$ obtained in the observation of point-like objects presented in [A to the case of extended objects, one can estimate whether a given galaxy could be significantly detected above the sky background.

It is thus concluded that a typical galaxy of $I=17$ would be detected about $60 \%$ of the times (i.e. 50 times on average during a 5 -year mission) with a $S / N>4$ using an area of $2 \times 2$ $\operatorname{arcsec}^{2}$ for the detection, as shown in Figure 1. According to our afore-mentioned statistical model, there are about 3 million galaxies brighter than this limit away from the Galactic plane (i.e. with $|b|>15$, where galaxy detection shouldn't be hampered by the high density of stars). It can thus be conservatively concluded that GAIA would be able to reliably detect at least 3 million galaxies, in agreement within a factor of two with the more optimistic estimation obtained in [8] following a different approach. Bright galaxies such as those appearing in [1] would thus typically be detected down to $d_{\text {det }} \simeq 400 \mathrm{Mpc}$ or $z_{\text {det }} \simeq 0.1$.

\section{GALAXY OBSERVATION VIA NUMERICAL SIMULATIONS}

In order to assess the angular resolution and the photometric accuracy obtainable from GAIA galaxy observations, complete simulation software based on HST WFPC2 images and generating representative all-mission sets of GAIA observations of the same field was developed. Among other things, this has also allowed to determine the optimal sample size and to test different stacking techniques. The Astro PSF resulting from different smearing effects was modelled using the tools provided in [7], whereas the expected CCD readnoise was calculated using the formulae given in [13] and conservatively assuming full CCD readout in all cases. 

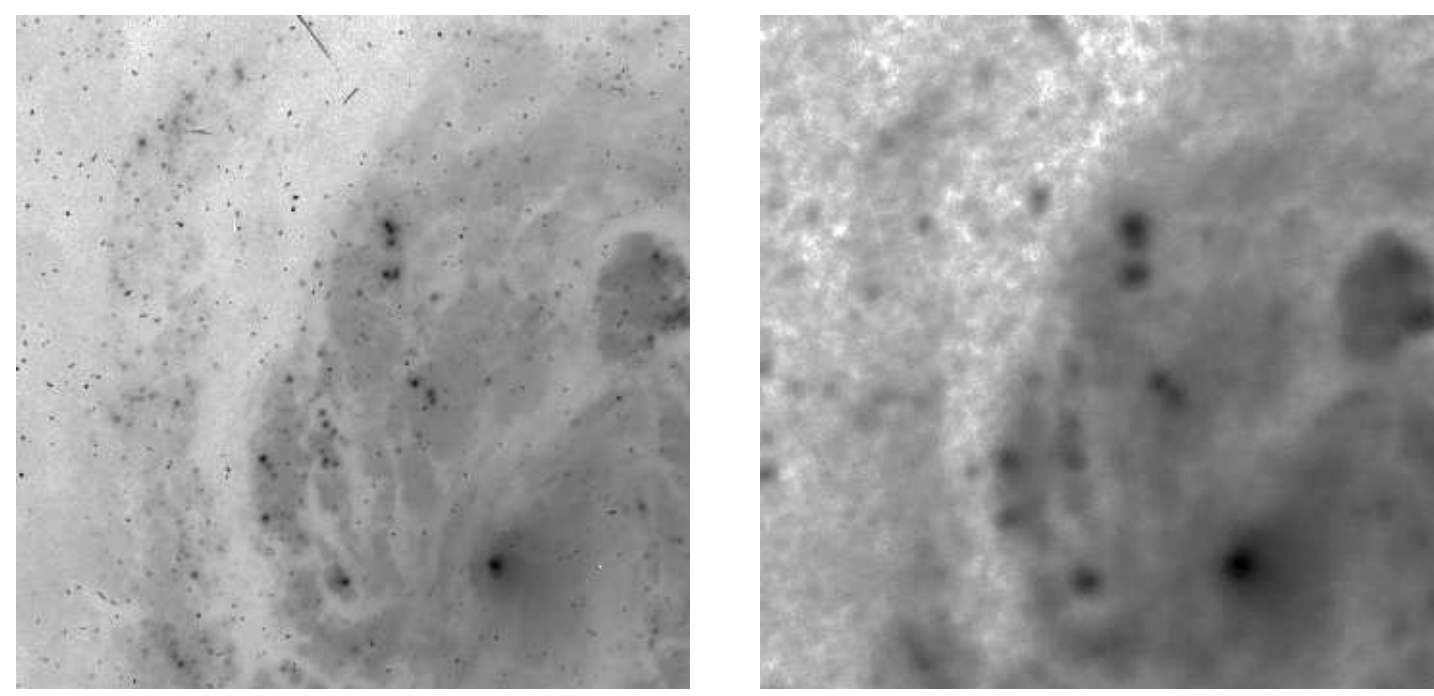

Fig. 2. HST Images and GAIA Flux Maps: HST WFPC2 $900 \mathrm{~s}$ image of the M100 Spiral Galaxy and GAIA BBP simulated flux map obtained from 50 observations, thus with a total effective exposure time of $45 \mathrm{~s}$. The side of both images is 16 arcmin.

The results of the simulations are shown in Figure 2, where the original HST WFPC2 $900 \mathrm{~s}$ image is compared with GAIA BBP flux map obtained from stacking of 50 observations of $0.9 \mathrm{~s}$ each, i.e. with an effective exposure time of $45 \mathrm{~s}$, and a sample size of $6 \times 4$ pixels, chosen as the best trade-off between the needs of angular resolution and photometric accuracy. Notwithstanding the great difference in exposure time, most details are still clearly visible in GAIA flux map, and the median photometric accuracy is of $\simeq 0.2 \mathrm{mag} / \operatorname{arcsec}^{2}$ at a median surface brightness of $\mu_{V} \simeq 20 \mathrm{mag} / \operatorname{arcsec}^{2}$, interestingly very similar to the predictions based on statistical formulae taking into account photon noise and readnoise.

The angular resolution achievable in GAIA BBP flux maps can instead be evaluated in Figure 3, where two bright HII regions with a separation of less than 0.5 arcsec are clearly resolved in GAIA flux map as well. More accurate estimates of the angular resolution based on model PSFs give an angular resolution as low as 0.35 arcsec, comparable to superb groundbased observing sites in the rare moments of excellent seeing. Galaxy observations could therefore be profitably carried out in Astro 2, where a sample size of $6 \times 4$ pixels is not in conflict with the baseline sample size of $6 \times 8$ pixels adopted for the observation of stars.

\section{SCIENTIFIC CASE}

The scientific case for a wide-angle high-resolution photometric (and possibly astrometric, even though the latter issue has not been studied in detail as yet) survey of bright galaxies is enormous. The high reliability catalogues extending down to low Galactic latitudes and high spatial resolution imaging of all sufficiently high surface brightness galaxies provided by GAIA will make up a remarkably vast, homogeneous and well-defined database that will have a tremendous impact at two levels: for statistical analysis of the photometric structure of the central regions of tens of thousands of well-resolved galaxies, and for the study of the large scale structure of the Local Universe from the spatial distribution of all detected galaxies. Detailed analysis of the inner luminosity profiles of a large sample of galaxies will define the 

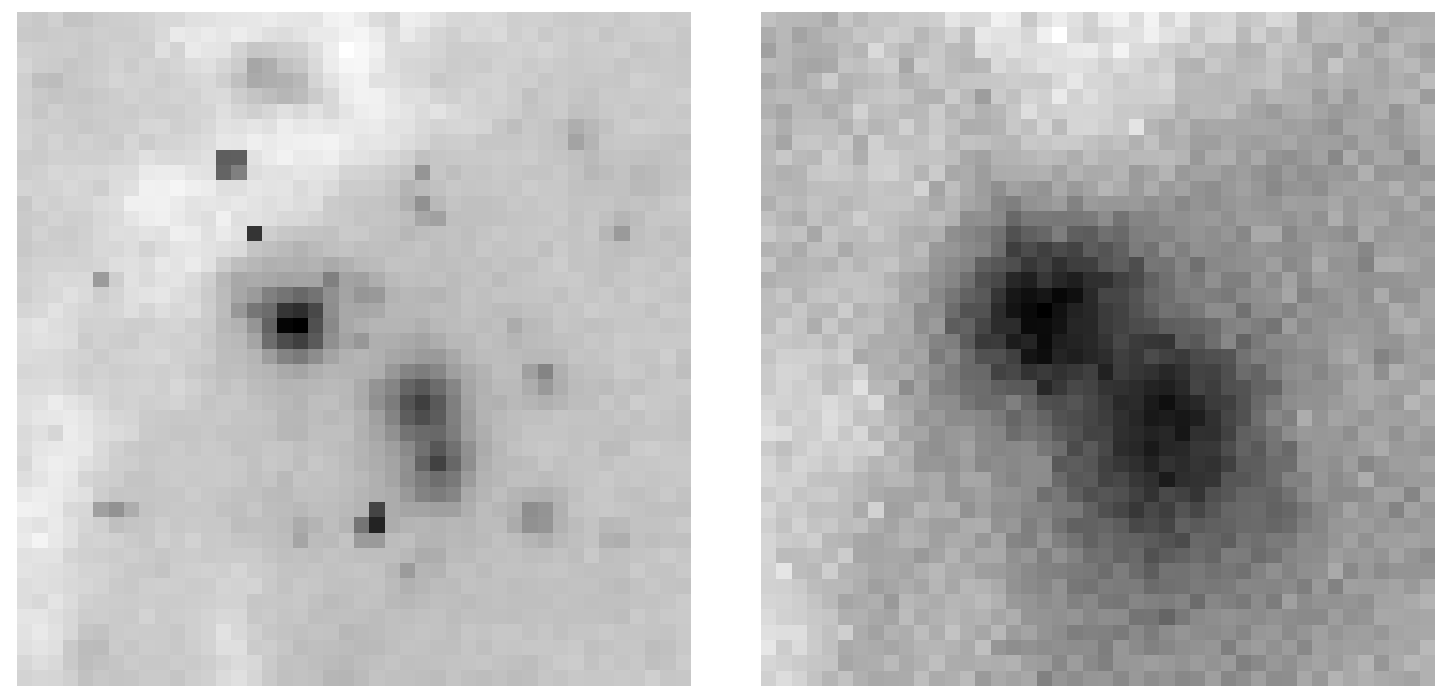

Fig. 3. Angular Resolution of GAIA Flux Maps: a small portion near the center of the same HST WFPC2 image and GAIA BBP flux map shown in Figure 2, showing two HII regions with a separation of about 0.5 arcsec.

true incidence of core structures and complex morphologies. Inner color gradients will map recent star formation and dust lanes, and central luminosity cusps may indicate massive black holes. Multi-colour and multi-epoch information will allow the identification of variable sources for dedicated follow-up by other telescopes. More in general, in conjunction with the proposed high-resolution survey for the detection of stars fainter than the nominal detection limit and the low-resolution survey of the sky background surface brightness, GAIA promises to yield the first uniform measurements of brightness gradients of the "real sky" at all spatial scales.

\section{MISSION PREPARATION, OPERATIONS AND TELEMETRY}

Several aspects of the picture which was outlined require careful definition. The galaxy detection process must be validated through the development of a dedicated algorithm, suitable for the reliable detection of faint extended objects on a bright complex background. One must then make sure that the Astro CCDs can be operated so that they can switch to the galaxy observation mode, possibly reading the full CCD, whenever a galaxy is detected. The opportunity of galaxy observations in the AF17 and in the SSM, where the much higher sensitivity and spectral resolution, respectively, would result in a much higher accuracy in surface photometry and astrophysical characterization of the observed galaxies, should be discussed. Finally, but perhaps most importantly, it must be evaluated how the required telemetry (on average 120 $\mathrm{kbits} / \mathrm{s} /$ band before compression, where most readout samples will carry little signal and thus allow efficient compression) can be accommodated within the overall telemetry budget.

\section{CONCLUSIONS}

The proposed GAIA Galaxy Survey will provide a nearly all-sky, multi-color and multi-epoch astrometric and photometric galaxy survey. In the framework of the present mission design, the feasibility, scientific case and optimization of such a survey were discussed. From both 
statistical considerations and numerical simulations it appears that galaxies could be reliably detected in the ASM 1 within square areas of $2 \times 2 \operatorname{arcsec}^{2}$ and observed in the Astro 2 BBP with a sample size of $6 \times 4$ pixels. The first choice should yield the highest number of detected galaxies without too may false detections, whereas the second one would provide the best trade-off between angular resolution, readnoise and telemetry. Under the present assumptions about the instrumental performance of the satellite payload, and provided some effort is put into its planning in the near future, the following measurement capabilities are expected from a 5-year mission:

- At least 3 million galaxies brighter than $I \simeq 17$ will be detected.

- All detected galaxies will be observed with a 0.35 arcsec angular resolution and an allmission accuracy in surface photometry of $0.2 \mathrm{mag} / \operatorname{arcsec}^{2}$ at $20.0 \mathrm{mag} / \operatorname{arcsec}^{2}$ in the $V$ band.

- Multi-color (in the 4-5 BBP broad bands) and multi-epoch ( $\simeq 50$ epochs) information will be available for all observed objects. White light and medium-band photometry could as well be obtained.

These outstanding measurement capabilities will result in a unique dataset providing stateof-the-art information on galaxy spatial distribution and surface photometry over a well-defined sample extending down to low Galactic latitudes, which is in turn expected to yield significant scientific results concerning the large-scale structure of the Local Universe and the multi-color photometric structure of galaxy innermost regions.

\section{References}

[1] de Vaucouleurs G., de Vaucouleurs A., Corwin H.G., Buta R.J., Paturel G. and Fouqué P. 1991, Third Reference Catalogue of Bright Galaxies, Springer

[2] ESA 2000, GAIA: Composition, Formation and Evolution of the Galaxy, Concept and Technology Study Report, ESA-SCI(2000) 4

[3] Høg E., Fabricius C., Knude J. and Makarov V.V. 1998, GAIA Surveys of Nebulae and Sky Background, Technical Report SAG_CUO_32

[4] Høg E., Fabricius C., Knude J. and Makarov V.V. 1999, Sky Survey and Photometry by the GAIA Satellite, Technical Report SAG_CUO_53 and Baltic Astronomy, 8, 25-56

[5] Høg E. 2001a, Photometric and imaging performance, Technical Report GAIA_CUO_91 and this volume

[6] Høg E. 2001b, Multi-colour photometry with GAIA of the diffuse sky background, Technical Report GAIA_CUO_92 and this volume

[7] Lindegren L. 1998, Point Spread Functions for GAIA including aberrations, Technical report GAIA_LL_25

[8] Lindegren L. 2000, Detection of faint galaxies with GAIA, Technical Report GAIA_LL_29

[9] Perryman M.A.C. et al. 2001, GAIA: Composition, Formation and Evolution of the Galaxy, A\&A, 369, 339-363

[10] Vaccari M. and Høg E. 1999a, Statistical Model of Galaxies, Technical Report GAIA_CUO_61, HTML version available at http://hal.pd.astro.it/ mattia/research/smog/

[11] Vaccari M. and Høg E. 1999b, Simulated GAIA Observations of Galaxies, Technical Report GAIA_CUO_69

[12] Vaccari, M. 2000, GAIA Galaxy Survey, Master Thesis, University of Padova. Available at http://hal.pd.astro.it/ mattia/research/ or upon request writing to vaccari@pd.astro.it

[13] Vannier M. 1998, Noise of GAIA Astro Instrument, Technical Report GAIA_MV_04 